\title{
Prevalence and Factors Associated With Atrial Fibrillation in Elderly Patients With Obstructive Sleep Apnea
}

Huanhuan Wang

Yan'an University

JianHua Li

Chinese PLA General Hospital

Yinghui Gao

Peking University International Hospital

Kaibing Chen

the affiliated Hospital of Gansu University of Chinese Medicine

Yan Gao

960th Hospital of PLA

JingJing Guo

Peking University People's Hospital

Min Shi

Chinese PLA General Hospital

Xiao Zou

Chinese PLA General Hospital

Weihao Xu

Chinese PLA General Hospital

LiBo Zhao

Chinese PLA General Hospital

Xiaofeng Su

Yan'an University

Yabin Wang

Chinese PLA General Hospital

Juan Liu

Chinese PLA General Hospital

$\mathrm{Hu} \mathrm{Xu}$

Chinese PLA General Hospital

Xiaoxuan Kong

Chinese PLA General Hospital

Junling Lin 
Beijing Chaoyang Hospital, Capital Medical University

\section{Xiaoshun Qian}

Chinese PLA General Hospital

Jiming Han

Yan'an University

Lin Liu ( $\square$ liulin715@qq.com )

Chinese PLA General Hospital

\section{Research Article}

Keywords: Obstructive sleep apnea, atrial fibrillation, elderly, prevalence, china

Posted Date: July 29th, 2021

DOl: https://doi.org/10.21203/rs.3.rs-701664/v1

License: (c) (i) This work is licensed under a Creative Commons Attribution 4.0 International License. Read Full License 


\section{Abstract}

Purpose: This study sought to identify the prevalence and factors associated with atrial fibrillation (AF) in elderly patients with obstructive sleep apnea (OSA) in China.

Methods: Between January 2015 and October 2017, we recruited 1285 elderly patients with OSA who underwent overnight polysomnography at sleep centers of multiple hospitals. They were assessed using 12-lead ECG or 24-hour dynamic ECG, and their baseline demographics, clinical characteristics, sleep parameters, and medical history were determined. Binary logistic regression analysis was used to investigate the factors related to $\mathrm{AF}$ in these elderly patients.

Results: The clinician classified $122(9.5 \%)$ patients as having AF. The prevalence of AF significantly

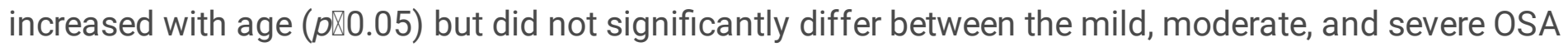
groups. Additionally, the prevalence of paroxysmal AF was $7.2 \%$ among the overall study population, and it increased with OSA severity or advanced age ( $p \bigotimes 0.05)$. Persistent AF was noted in $2.3 \%$ participants, and the prevalence also increased with age. The logistic regression analysis showed that age $(O R=1.054$, $95 \% \mathrm{Cl}: 1.027-1.018, p \varangle 0.001)$, history of drinking (OR=1.752, 95\% Cl: 1.070-2.867, $p \varangle 0.05)$, chronic heart disease (OR=1.778, 95\%Cl: 1.156-2.736, $p \bowtie 0.01)$, diabetes mellitus (OR=1.792, 95\%Cl: 1.183-2.713, $p \rrbracket$ $0.01)$, and cardiac dysfunction $(\mathrm{OR}=2.373,95 \% \mathrm{Cl}=1.298-4.337, p \otimes 0.01)$ were relevant to $\mathrm{AF}$ among participants with OSA.

Conclusion: The prevalence of AF is significantly common in elderly patients with OSA. Age, history of drinking, chronic heart disease, diabetes mellitus, and cardiac dysfunction are independently related to AF in these patients.

\section{Introduction}

Obstructive sleep apnea (OSA), the most common type of sleep apnea, is an independent risk factor for cardiovascular diseases and all-cause mortality ${ }^{[1-3]}$. Moreover, patients with OSA were frequently diagnosed with hypotension ${ }^{[4]}$, diabetes mellitus $(\mathrm{DM})^{[5]}$, and stroke ${ }^{[6]}$. Several clinical studies have revealed that OSA is highly prevalent $(60-90 \%)$ in patients with atrial fibrillation $(\mathrm{AF})^{[7-}$ 9]. OSA promotes the occurrence and recurrence of $A F$, which is the most common clinically observed form of arrhythmia, due to the repetitive cycles of intermittent hypoxia causing an imbalance of cardiac autonomic modulation ${ }^{[9]}$. Moreover, older patients with OSA are more prone to developing $\mathrm{AF}^{[10-11]}$.

The prevalence of OSA among patients with AF, including those who underwent catheter ablation has been reported; however, the prevalence and factors associated with AF in OSA patients are not clearly studied. Therefore, we performed this large-scale, multicenter, cross-sectional study to determine the prevalence and risk factors of AF among elderly patients with OSA.

\section{Methods}




\section{Study Population}

Initially, 1290 patients were recruited from the PLA General Hospital, Peking University International Hospital, Peking University People's Hospital, Beijing Chaoyang Hospital, 960th Hospital of PLA, and the affiliated Hospital of Gansu University of Chinese Medicine between January 2015 and October 2017. Our inclusion criteria were as follows:1) age $\geq 60$ years; 2 ) diagnosis of OSA by a clinician based on overnight polysomnography (PSG); 3) assessment using 12-lead ECG or 24-hour dynamic ECG. The exclusion criteria were as follows: prior history of valvular atrial fibrillation (5/1290), hyperthyroidism, and severe electrolyte disorder. Ultimately, 1285 elderly patients with OSA were included in the analysis.

The ethics committee of PLA General Hospital approved the study (S2019-352-01).

\section{Baseline Evaluation}

Demographic data and clinical characteristics of all patients were collected by the researchers, including sex, age, nationality, height, weight, body mass index (BMI), personal history, blood pressure, history of smoking, and history of drinking, as well as data on sleep parameters, blood index included TG, Creatinine, Blood glucose, RBC, WBC, PLTs, HB, and medical history. These data were used to screen patients who met the inclusion and exclusion criteria in this study.

\section{PSG Examination}

PSG is the gold standard of OSA diagnosis. All participants underwent full overnight PSG at the sleep center of different hospitals. The sleep parameters of all patients were recorded using a laboratory-based PSG instrument (Compumedics, Melbourne, Australia), including electroencephalography

(EEG), electrooculography (EOG), electrocardiography (ECG), nasal-oral airflow, chest and abdominal wall motion, arterial oxygen saturation, and body position ${ }^{[12]}$. PSG records were staged manually according to standard criteria. Apnea was defined as the continuous cessation of airflow for more than $10 \mathrm{~s}$, whereas hypopnea was defined as a $30 \%$ or greater drop in flow for 10 s or longer associated with $\geq 4 \%$ oxygen desaturation (obstructive sleep apnea if respiratory efforts were present) ${ }^{[13]}$. The apnea hypopnea index (AHI) was defined as the number of apnea and hypopnea events per hour during sleep. OSA severity was defined according to AHI based on the criteria of the American Academy of Sleep Medicine as follows ${ }^{[13]}$ : mild OSA, $5 \leq \mathrm{AHI}<15$; moderate OSA, $15 \leq \mathrm{AHI}<30$; and severe OSA, $\mathrm{AHI} \geq 30$.

\section{ECG Examination}

For all patients enrolled in the study who were assessed using12-lead ECG or 24-hour dynamic ECG, the clinical diagnosis of AF was based on the ACC/AHA/ESC 2016 guidelines $^{[14]}$. The key electrocardiographic findings of AF are a loss of $\mathrm{P}$ waves and replacement by fibrillatory waves; erratic activation of the ventricles resulting in an irregular, rapid heart rate (usually 90 to $170 \mathrm{bpm}$ ); and a narrow QRS complex, unless other conduction abnormalities coexist ${ }^{[15]}$.

\section{Statistical Analysis}


All data were analyzed using SPSS version 20.0 (SPSS Inc, Chicago, IL, USA). Metrological data were first tested for normality and homogeneity of variance. Normally distributed metrological data are expressed as the mean \pm standard deviation (SD), and one-way analysis of variance or the $t$ test was used for comparisons between groups. Metrological data that did not meet the criterion for normal distribution are expressed as the median (interquartile range), and non-parametric tests were used for comparisons between groups. Count data are expressed as percentage (\%), and chi-square tests were used for comparisons between groups. Multivariate analyses were performed using binary logistic regression, the results of which are expressed as odds ratio (OR) with 95\% confidence interval (Cl).

\section{Results}

\section{Baseline Characteristics}

A total of 1285 OSA patients were included for data analysis, including 305 (23.7\%) with mild OSA, 385 (30\%) with moderate OSA, and $595(46.3 \%)$ with severe OSA.

Patients with severe OSA were more likely to be male and had higher BMI and systolic blood pressure (SBP), but patients with mild OSA were older. In terms of serological indices, the concentration of TG and creatinine and the white blood cell (WBC) count were higher in patients with severe OSA. The prevalence of hypertension, hyperlipidemia, coronary heart disease (CHD), cerebrovascular disease, $\mathrm{DM}$, and AF was higher in this population ( $p \varangle 0.05$, Table 1 ). Participants diagnosed with AF were found to be older, had a higher SBP, and accounted for a higher proportion of smokers and drinkers. The scores for sleep parameters including $\mathrm{AHI}$ and oxygen desaturation index(ODI) were higher in $\mathrm{AF}$ than non-AF patients, but $\mathrm{LSpO}_{2}$ was lower in the AF group. TG concentration was lower and creatinine concentration was higher in $\mathrm{AF}$ than non-AF patients. Additionally, those with $\mathrm{AF}$ had a higher proportion of CHD, cerebrovascular disease, carotid atherosclerosis, DM, peripheral vascular disease(PVD), chronic obstructive pulmonary disease, chronic kidney disease(CKD), and cardiac dysfunction ( $p \bowtie 0.05)$; other indexes did not significantly differ between the two groups (Table 2).

\section{Prevalence of AF in elderly patients with OSA}

There were 122 (9.5\%) patients with clinically classified AF. Of these, the prevalence of AF in males was higher than that in females: $10.2 \%$ in males vs. $8.4 \%$ in females. Moreover, the prevalence of AF significantly increased with age: $7.0 \%$ in patients aged $60-70$ years vs. $14.9 \%$ in patients aged $71-96$ years. The prevalence of AF tended to increase with the severity of OSA, but there was no significant difference between the three groups (Figure 1). The prevalence of paroxysmal AF was $7.2 \%$ among the total study population, and it significantly increased with OSA severity or advanced age ( $p \square$ 0.05). Similarly, the prevalence of $A F$ in males was higher than that in females (7.7\% vs. $6.5 \%$; Figure 2$)$. Furthermore, $2.3 \%$ participants were found to have persistent $A F$, and although the prevalence also increased with age, it did not significantly differ between the mild, moderate, and severe OSA groups (Figure 3). 


\section{Factors associated with AF in elderly patients with OSA}

Age (OR=1.055, 95\% Cl: 1.028-1.082), history of drinking(OR=1.795, 95\% Cl: 1.102-2.923), CHD (OR=1.834, 95\%Cl: 1.196-2.812), DM (OR=1.756, 95\%Cl: 1.163-2.653), and cardiac dysfunction $(\mathrm{OR}=3.033,95 \% \mathrm{Cl}: 1.757-5.234)$ were independently related to $\mathrm{AF}$ in participants after adjustments for smoking, SBP, AHI, ODI, LSpO2, TG, creatinine, cerebrovascular disease, carotid atherosclerosis, PVD, chronic obstructive pulmonary disease, and CKD (Table 3).

$\mathrm{D}(\mathrm{OR}=1.834,95 \% \mathrm{Cl} 1.196-2.812), \mathrm{DM}(\mathrm{OR}=1.756,95 \% \mathrm{Cl} 1.163-2.653)$ and cardiac dysfunction $(\mathrm{OR}=3.033,95 \% \mathrm{Cl} 1.757-5.234)$ were independently related to $\mathrm{AF}$ in participants after adjustments for smoking, SBP, AHI, ODI, LSaO2, TG, creatinine, cerebrovascular disease, carotid atherosclerosis, PVD, COPD, CKD (Table 3).

Table1. Characteristics of study participants 
Indictor

Mild OSA

$(n=305)$
Moderate OSA

$(n=385)$
Severe OSA

$(n=595)$
$P$

value

Demographics

\begin{tabular}{|c|c|c|c|c|}
\hline Age, y & $67.00 \rrbracket 63.50 \rrbracket 72.00 \rrbracket$ & $66.00 \rrbracket 62.00 \rrbracket 72.00 \rrbracket$ & $65.00 \rrbracket 62.00 \otimes 71.00 \rrbracket$ & 0.004 \\
\hline Male & $168(55.1)$ & $231(60.0)$ & $395(66.4)$ & 0.003 \\
\hline Height (cm) & $165.18 \pm 8.02$ & $165.76 \pm 7.92$ & $166.48 \pm 7.83$ & 0.056 \\
\hline Weight (kg) & $69.10 \pm 11.77$ & $71.30 \pm 11.69$ & $77.65 \pm 12.95$ & 0.001 \\
\hline 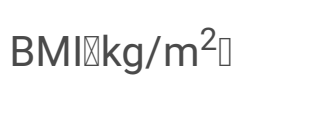 & $25.15 \rrbracket 22.66 \rrbracket 27.47 \rrbracket$ & $25.76 \rrbracket 23.63 \rrbracket 28.08 \rrbracket$ & $27.64 \llbracket 25.25 \llbracket 30.80 \rrbracket$ & $\begin{array}{l}\square \\
0.001\end{array}$ \\
\hline Smoking, n (\%) & $59(19.3)$ & $81(21.0)$ & $152(25.5)$ & 0.070 \\
\hline Drinking, n (\%) & $30(9.8)$ & $42(10.9)$ & $85(14.3)$ & 0.100 \\
\hline $\mathrm{SBP}(\mathrm{mmHg})$ & $\begin{array}{l}130.00 \rrbracket 120.00 \rrbracket \\
142.00 \rrbracket\end{array}$ & $\begin{array}{l}130.00 \rrbracket 121.50 \rrbracket \\
147.00 \rrbracket\end{array}$ & $\begin{array}{l}135.00 \rrbracket 126.00 \rrbracket \\
147.00 \rrbracket\end{array}$ & 0.034 \\
\hline $\mathrm{DBP}(\mathrm{mmHg})$ & $76.00 \otimes 70.00 \rrbracket 80.50 \rrbracket$ & $75.00 \rrbracket 70.00 \rrbracket 82.00 \rrbracket$ & $76.00 \otimes 70.00 \rrbracket 84.00 \rrbracket$ & 0.156 \\
\hline \multicolumn{5}{|l|}{ Sleep parameters } \\
\hline $\mathrm{AHI}$ & $9.30 \rrbracket 6.70 \rrbracket 11.90 \rrbracket$ & $21.70 \rrbracket 18.10 \rrbracket 25.90 \rrbracket$ & $47.80 \rrbracket 37.30 \rrbracket 60.10 \rrbracket$ & $\begin{array}{l}\square \\
0.001\end{array}$ \\
\hline ODI & $8.30 \rrbracket 4.65 \rrbracket 11.70 \rrbracket$ & $17.70 \rrbracket 12.25 \rrbracket 23.35 \rrbracket$ & $41.10 \rrbracket 29.50 \rrbracket 54.20 \rrbracket$ & $\begin{array}{l}0.001 \\
0.001\end{array}$ \\
\hline TST(h) & $7.10 \otimes 6.27 ه 7.38 \rrbracket$ & $7.11 \rrbracket 6.31 \otimes 7.73 \rrbracket$ & 7.17ه6.21ه7.79® & 0.140 \\
\hline MSp02 & $94.00 \rrbracket 92.00 \rrbracket 95.20 \rrbracket$ & $94.00 \rrbracket 92.00 \rrbracket 95.00 \rrbracket$ & $93.00 \otimes 91.00 \otimes 94.70 \rrbracket$ & $\begin{array}{l}\square \\
0.001\end{array}$ \\
\hline LSpO2 & $84.00 \rrbracket 80.00 \rrbracket 87.00 \rrbracket$ & $81.00 \rrbracket 76.00 \rrbracket 85.00 \rrbracket$ & $75.00 \rrbracket 65.00 \rrbracket 82.00 \rrbracket$ & $\begin{array}{l}\square \\
0.001\end{array}$ \\
\hline
\end{tabular}

blood index

\begin{tabular}{|c|c|c|c|c|}
\hline $\mathrm{TG}(\mathrm{mmol} / \mathrm{l})$ & $1.36 \rrbracket 1.00 \rrbracket 1.88 \rrbracket$ & $1.30 \rrbracket 0.96 \rrbracket 1.81 \rrbracket$ & $1.44 \rrbracket 1.04 \rrbracket 1.96 \rrbracket$ & 0.028 \\
\hline Creatinine $(\mu \mathrm{mol} / \mathrm{L})$ & $68.30 \otimes 59.5 \rrbracket 78.00 \rrbracket$ & $73.00 \rrbracket 62.63 \rrbracket 85.00 \rrbracket$ & $74.40 \rrbracket 63.4 \llbracket 86.30 \rrbracket$ & $\begin{array}{l}\square \\
0.001\end{array}$ \\
\hline $\begin{array}{l}\text { Blood glucose } \\
(\mathrm{mmol} / \mathrm{L})\end{array}$ & $5.59 \llbracket 5.01 \rrbracket 6.43 \rrbracket$ & $5.66 \rrbracket 5.06 \rrbracket 6.48 \rrbracket$ & $5.71 \rrbracket 5.09 \rrbracket 6.55 \rrbracket$ & 0.259 \\
\hline $\mathrm{RBC} \otimes 10^{12} / \mathrm{L} \rrbracket$ & $4.49 \llbracket 4.17 \rrbracket 4.83 \rrbracket$ & $4.47 \rrbracket 4.15 \rrbracket 4.78 \rrbracket$ & $4.51 \rrbracket 4.22 \rrbracket 4.85 \rrbracket$ & 0.280 \\
\hline WBC $\left(10^{9} / \mathrm{L}\right)$ & $6.08 \bowtie 5.09 \otimes 7.13 \rrbracket$ & $6.01 \rrbracket 5.06 \bigotimes 7.06 \rrbracket$ & $6.48 \rrbracket 5.51 \rrbracket 7.42 \rrbracket$ & $\begin{array}{l}\square \\
0.001\end{array}$ \\
\hline
\end{tabular}




\begin{tabular}{|c|c|c|c|c|}
\hline PLTs $\left(10^{9} / \mathrm{L}\right)$ & $\begin{array}{l}203.00 \rrbracket 168.00 \rrbracket \\
239.00 \rrbracket\end{array}$ & $\begin{array}{l}201.67 \rrbracket 169.83 \rrbracket \\
236.00 \rrbracket\end{array}$ & $\begin{array}{l}205.33 \rrbracket 174.92 \rrbracket \\
238.00 \rrbracket\end{array}$ & 0.174 \\
\hline 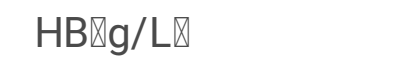 & $139.20 \pm 17.53$ & $138.22 \pm 17.72$ & $139.10 \pm 18.55$ & 0.887 \\
\hline \multicolumn{5}{|l|}{ Medical history, n (\%) } \\
\hline Hypertension & $190(62.3)$ & $231(60.0)$ & $411(69.1)$ & 0.009 \\
\hline Hyperlipidemia & $96(31.5)$ & $102(26.5)$ & $160(26.9)$ & 0.270 \\
\hline GERD & $10(3.3)$ & $20(5.2)$ & $12(2.0)$ & 0.024 \\
\hline $\mathrm{CHD}$ & $55(18.0)$ & $97(25.2)$ & $152(25.5)$ & 0.030 \\
\hline $\begin{array}{l}\text { Cerebrovascular } \\
\text { disease }\end{array}$ & $48(15.7)$ & $64(16.6)$ & $115(19.3)$ & 0.333 \\
\hline $\begin{array}{l}\text { Carotid } \\
\text { atherosclerosis }\end{array}$ & $96(31.5)$ & $99(25.7)$ & $135(22.7)$ & 0.017 \\
\hline DM & $59(19.3)$ & $93(24.2)$ & $165(27.7)$ & 0.021 \\
\hline Liver disease & $11(3.6)$ & $26(6.8)$ & $37(6.2)$ & 0.171 \\
\hline $\begin{array}{l}\text { Connective tissue } \\
\text { disease }\end{array}$ & $2(0.7)$ & $4(1.0)$ & $6(1.0)$ & 0.834 \\
\hline Peptic ulcer & $7(2.3)$ & $8(2.1)$ & $6(1.0)$ & 0.253 \\
\hline PVD & $13(4.3)$ & $21(5.5)$ & $29(4.9)$ & 0.771 \\
\hline Solid tumor & $15(4.9)$ & $19(4.9)$ & $17(2.9)$ & 0.166 \\
\hline COPD & $25(8.2)$ & $31(8.1)$ & $36(6.1)$ & 0.358 \\
\hline CKD & $6(2.0)$ & $15(3.9)$ & $31(5.2)$ & 0.064 \\
\hline Cardiac dysfunction & $19(6.2)$ & $26(6.8)$ & $40(6.7)$ & 0.953 \\
\hline \multicolumn{5}{|l|}{$\mathrm{AF}$} \\
\hline Paroxysmal AF & $10(3.3)$ & $28(7.3)$ & $55(9.2)$ & 0.008 \\
\hline Persistent AF & $11(3.6)$ & $6(1.6)$ & $12(2.0)$ & \\
\hline \multicolumn{5}{|c|}{$\begin{array}{l}\text { BMI: body mass index; SBP: systolic blood pressure; DBP: diastolic blood pressure; AHI: the apnea- } \\
\text { hypopnea index; ODI: the oxygen desaturation index; TST: total sleep time; MSpO2: the mean pulse } \\
\text { oxygen saturation; LSpO2: the lowest pulse oxygen saturation; TG: triglyceride; RBC: red blood cell; WBC: } \\
\text { white blood cell; PLTs: platelets; HB: hemoglobin; GERD } ₫ \text { gastroesophageal reflux disease; CHD: coronary } \\
\text { heart disease; DM: diabetes mellitus; PVD: peripheral vascular disease; COPD: chronic obstructive } \\
\text { pulmonary disease; CKD: chronic kidney disease; AF: atrial fibrillation. }\end{array}$} \\
\hline
\end{tabular}


Table 2. Characteristics of AF and non-AF participants 
Indicator

Non-AF group

AF group

$P$-value

$(n=1163)$

$(n=122)$

Demographics

\begin{tabular}{|c|c|c|c|}
\hline Age, y & $66.00 \rrbracket 62.00 \rrbracket 71.00 \rrbracket$ & $69.60 \rrbracket 64.00 \rrbracket 77.00 \rrbracket$ & $\varangle 0.001$ \\
\hline Male & 713ه61.3》 & $81 \otimes 10.2 \rrbracket$ & 0.271 \\
\hline Height (cm) & $165.86 \pm 7.89$ & $166.89 \pm 8.08$ & 0.168 \\
\hline Weight (kg) & $73.51 \pm 12.74$ & $74.99 \pm 13.75$ & 0.229 \\
\hline $\mathrm{BM} \otimes \mathrm{kg} / \mathrm{m}^{2} \mathrm{\square}$ & $26.49 \rrbracket 24.03 \rrbracket 29.05 \rrbracket$ & $26.70 \rrbracket 24.22 \rrbracket 30.65 \rrbracket$ & 0.255 \\
\hline Smoking, n (\%) & $250 \rrbracket 21.5 \rrbracket$ & $42 \bowtie 34.4 \rrbracket$ & 0.001 \\
\hline Drinking, n (\%) & 128ه11.0》 & $29 \llbracket 23.8 \rrbracket$ & $\varangle 0.001$ \\
\hline $\mathrm{SBP}(\mathrm{mmHg})$ & $131.00 \otimes 124.00 \otimes 144.00 \rrbracket$ & $140.00 \otimes 125.75 \bigotimes 160.00 \rrbracket$ & 0.002 \\
\hline DBP (mmHg) & $76.00 \otimes 70.00,82.00 \rrbracket$ & $77.50(70.00,85.25)$ & 0.163 \\
\hline
\end{tabular}

Sleep parameters

\begin{tabular}{|c|c|c|c|}
\hline $\mathrm{AHI}$ & $27.20 \rrbracket 15.1 \rrbracket 45.60 \rrbracket$ & $32.20 \rrbracket 20.60 \rrbracket 46.83 \rrbracket$ & 0.044 \\
\hline ODI & $22.20 \rrbracket 10.70 \rrbracket 40.50 \rrbracket$ & $26.95 \rrbracket 17.08 \rrbracket 43.00 \rrbracket$ & 0.014 \\
\hline TST(h) & $7.11(6.23,7.60)$ & $7.20(6.39,7,71)$ & 0.304 \\
\hline MSpO2 & $93.00 \rrbracket 91.80 \rrbracket 95.00 \rrbracket$ & $93.00 \otimes 91.00 \otimes 95.00 \rrbracket$ & 0.112 \\
\hline LSpO2 & $80.00 \rrbracket 72.00 \rrbracket 85.00 \rrbracket$ & $77.00 \rrbracket 68.00 \rrbracket 83.00 \rrbracket$ & 0.005 \\
\hline \multicolumn{4}{|l|}{ blood index } \\
\hline $\mathrm{TG}(\mathrm{mmol} / \mathrm{l})$ & $1.39(1.02,1.92)$ & $1.32(0.93,1.72)$ & 0.039 \\
\hline Creatinine $(\mu \mathrm{mol} / \mathrm{L})$ & $72.00(61.75,83.00)$ & $76.00(66.15,88.83)$ & 0.003 \\
\hline Blood glucose $(\mathrm{mmol} / \mathrm{L})$ & $5.67(5.06,5.67)$ & $5.55(5.03,6.69)$ & 0.833 \\
\hline 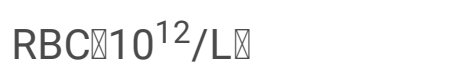 & $4.50 \pm 0.56$ & $4.45 \pm 0.60$ & 0.393 \\
\hline WBC $\left(10^{9} / \mathrm{L}\right)$ & $6.24(5.26,7.19)$ & $6.48(5.48,5.51)$ & 0.141 \\
\hline $\operatorname{PLTs}\left(10^{9} / \mathrm{L}\right)$ & $204.00(171.00,238.5)$ & $201.00(168.00,235.25)$ & 0.403 \\
\hline $\mathrm{HB} \bowtie \mathrm{g} / \mathrm{L} \rrbracket$ & $137.95 \pm 17.41$ & $137.07 \pm 17.52$ & 0.594 \\
\hline \multicolumn{4}{|l|}{ Medical history, n (\%) } \\
\hline Hypertension & $750 \otimes 64.5 \rrbracket$ & $82 \rrbracket 67.2 \rrbracket$ & 0.549 \\
\hline Hyperlipidemia & $318 \otimes 27.3 \rrbracket$ & 40囚32.8囚 & 0.202 \\
\hline
\end{tabular}




\begin{tabular}{|c|c|c|c|}
\hline GERD & $38 \bowtie 3.3 \rrbracket$ & $4 \llbracket 3.3 \rrbracket$ & 0.995 \\
\hline $\mathrm{CHD}$ & $247 \rrbracket 21.2 \rrbracket$ & $57 \rrbracket 46.7 \rrbracket$ & $\nabla 0.001$ \\
\hline Cerebrovascular disease & 194ه16.7凶 & $33 \llbracket 27.0 \rrbracket$ & 0.004 \\
\hline Carotid atherosclerosis & $279 \rrbracket 24.0 \rrbracket$ & $51 \rrbracket 41.8 \rrbracket$ & $\otimes 0.001$ \\
\hline DM & $267 \rrbracket 23.0 \rrbracket$ & $50 \rrbracket 41.0 \rrbracket$ & $\otimes 0.001$ \\
\hline Liver disease & $65 \rrbracket 5.6 \rrbracket$ & $9 \bigotimes 7.4 \rrbracket$ & 0.420 \\
\hline Connective tissue disease & $10 \otimes 0.9 \rrbracket$ & $2 \otimes 1.6 \rrbracket$ & 0.721 \\
\hline Peptic ulcer & 16ه1.4囚 & $5 \llbracket 4.1 \rrbracket$ & 0.060 \\
\hline PVD & $52 \rrbracket 4.5 \rrbracket$ & 11凶9.0ه & 0.027 \\
\hline Solid tumor & $44 \rrbracket 3.8 \rrbracket$ & $7 \rrbracket 5.7 \rrbracket$ & 0.419 \\
\hline COPD & $72 \rrbracket 6.2 \rrbracket$ & $20 \rrbracket 16.4 \rrbracket$ & $\nabla 0.001$ \\
\hline CKD & $41 \otimes 3.5 \rrbracket$ & 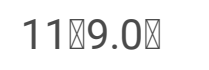 & 0.007 \\
\hline Cardiac dysfunction & $59 \llbracket 5.1 \rrbracket$ & $26 \rrbracket 21.3 \rrbracket$ & $\otimes 0.001$ \\
\hline
\end{tabular}

BMI: body mass index; SBP: systolic blood pressure; DBP: diastolic blood pressure; AHI: the apneahypopnea index; ODI: the oxygen desaturation index; TST: total sleep time; MSpO2: the mean pulse oxygen saturation; LSp02: the lowest pulse oxygen saturation; TG: triglyceride; RBC: red blood cell; WBC: white blood cell; PLTs: platelets; HB: hemoglobin; GERD $\llbracket$ gastroesophageal reflux disease; CHD: coronary heart disease; DM: diabetes mellitus; PVD: peripheral vascular disease; COPD: chronic obstructive pulmonary disease; CKD: chronic kidney disease; AF: atrial fibrillation.

Table.3 Factors associated with AF in elderly patients with OSA derived from logistic regression with forward stepwise regressive method

\begin{tabular}{llllll} 
& B & S.E. & Wals & P & OR $(95 \mathrm{Cl})$ \\
\hline Age & 0.053 & 0.013 & 16.117 & 0.000 & $1.055(1.028-1.082)$ \\
\hline Drinking & 0.585 & 0.249 & 5.531 & 0.019 & $1.795(1.102-2.923)$ \\
\hline CHD & 0.607 & 0.218 & 7.743 & 0.005 & $1.834(1.196-2.812)$ \\
\hline DM & 0.563 & 0.210 & 7.166 & 0.007 & $1.756(1.163-2.653)$ \\
\hline Cardiac dysfunction & 1.109 & 0.278 & 15.870 & 0.000 & $3.033(1.757-5.234)$
\end{tabular}

CHD: coronary heart disease; DM: diabetes mellitus. 


\section{Discussion}

The prevalence of $A F$ is reported to be approximately $7.6 \%$ in patients with suspected OSA, and it increases with the severity of OSA ${ }^{[16]}$. However, the prevalence in elderly patients with OSA has not been reported. We found that the prevalence of AF was $9.5 \%$ in elderly patients with OSA, and it tended to increase with the severity of OSA, but there was no significant difference between mild, moderate, and severe OSA groups. We also found that the prevalence of paroxysmal and persistent AF was $7.2 \%$ and $2.3 \%$ in this study population, respectively. The prevalence of paroxysmal AF increased with the OSA severity. Nobuaki ${ }^{[8]}$ et al. reported the prevalence of moderate-severe SA was up to $72 \%$ in patients with $A F$, and the recurrence of $A F$ after radiofrequency ablation was significantly reduced by treatment for $\mathrm{OSA}^{[7-8,17]}$. Therefore, it is important to study the mechanism of action common to both.

OSA is defined as the partial or complete obstruction of the superior respiratory airway causing repeated dyspnea during sleep at night, along with intermittent hypoxemia, hypercapnia, and intrathoracic pressure changes ${ }^{[8,18]}$. The nocturnal hypoxemia in patients with OSA prolongs the atrial refractory period and slows atrial conduction, increasing the heterogeneity of the cardiac conduction system. Secondly, repeated apnea at night can promote sympathetic activation and increase the vagal tone, causing arrhythmia or worsening it ${ }^{[17,19]}$. Stroke, Transient Ischemic Attack(TIA), and systemic embolism are the most serious consequences for $A F$, which significantly increase the mortality and disability rate ${ }^{[20]}$. Advanced age is a risk factor for both OSA and AF and is associated with dysfunctional and impaired immune regulation ${ }^{[10-11]}$. Yao ${ }^{[10]}$ et al. found that the prevalence of AF increases with age, especially in older populations( $\geq 60$ years). Consistent with the results of the above studies, we found a $5.4 \%$ increase in the risk of $\mathrm{AF}$ among patients for each additional year of age. Muscle relaxation and muscle strength decline could also be contributing factors in the elderly population. A decrease in the upper airway muscle tone can lead to upper airway obstruction, increasing the prevalence of OSA in elderly people ${ }^{[21-22]}$. The routine examination of OSA patients generally excludes 12-lead ECG or 24-hour dynamic ECG; therefore, the possibility of AF in OSA patients is overlooked. We should focus on the screening of AF in elderly patients with OSA to prevent the development of OSA-related cardiovascular and cerebrovascular diseases.

A history of drinking significantly contributes to AF occurrence. Drinking is reported to be a definite risk factor for new-onset AF and an independent predictor for recurrence of AF. A meta-analysis elaborated that moderate-severity levels of alcohol consumption are associated with an increase in AF risk, with each 1 SD per day consumed, by approximately $8 \%{ }^{[23]}$. Takigawa ${ }^{[6]}$ et al. demonstrated that the recurrence rate of $\mathrm{AF}$ after initial catheter ablation is higher in patients who consumed alcohol than in those who did not (41.9\% VS 34.1\%). Metabolites of alcohol have direct cardiotoxic effects and indirectly influence cardiac function by leading to disordered breathing during sleep, obesity, or nervous system diseases. Drinking affects the atrial electrophysiological properties, prolonging the intra-atrial and inter-atrial conduction, shortening the right-atrial effective refractory period, and increasing the dispersion of refractoriness, all leading to cardiac electrical remodeling and AF occurrence ${ }^{[24]}$. Given that the 
relationship between AF and OSA is well-documented, older OSA patients with binge drinking behaviors are highly prone to AF.

The risk of developing $A F$ is 1.792-fold higher in DM patients than in those without $D M$, indicating that DM is another factor related to AF development and also closely related to OSA. Lidia ${ }^{[25]}$ et al. showed that the risk of $A F$ increases by $32 \%$ in patients with DM. Similarly, Papazoglou ${ }^{[26]}$ et al. reported the DM-associated increase in AF risk of $40 \%$. Huang ${ }^{[27]}$ et al. revealed that the prevalence of DM is $16.8 \%$ in nonvalvular AF patients. Zhang ${ }^{[28]}$ et al. reported the prevalence of OSA as $60 \%$ in hospitalized patients with type $2 \mathrm{DM}$. DM can affect the prognosis of AF patients, and OSA is closely related to insulin resistance or metabolic disorders. OSA increases sympathetic activity, disrupting the hypothalamus-pituitary-adrenal axis, thereby increasing the risk of metabolic disorders in individuals with OSA. Secondly, intermittent hypoxia in OSA causes oxidative stress, which can directly and indirectly promote inflammation, leading to insulin resistance and impaired beta cell function, setting the stage for $D^{[29]}$. Given the close association among OSA, DM, and AF, the prevalence of $A F$ in patients with $D M$ patients was higher than that in patients without $D M$ in this study.

Cardiovascular diseases including $\mathrm{CHD}$ and cardiac dysfunction were associated with AF among participants. The repeated hypoxemia and hypercapnia in OSA lead to oxidative stress and inflammation. Reactive oxygen species activate nuclear factor-kappa B, which leads to increased production of Creactive protein, tumor necrosis factor-a, and interleukin-6, along with adhesion molecules such as $\mathrm{E}$ selectin, CD15, and CD32 that can cause endothelial damage, CHD, heart failure. And hypoxemia of OSA can decrease the oxygen of cardiomyocytes and cause systolic and diastolic dysfunction ${ }^{[2]}$. CHD and cardiac dysfunction commonly result in abnormal autonomic modulation to affect the stability of cardiac electrophysiology and cardiac pump function to promote the development of $\mathrm{AF}^{[30]}$. In summary, OSA was found to be an independent risk factor for cardiovascular diseases involving cardiac autonomic dysfunction. The hypoxemia in OSA causes sympathetic excitation and increases vagal tone, which could be associated with AF.

This study was a multicenter, large-sample study, and we have attempted to include as many relevant factors as possible in our analysis. However, there were a few limitations that the study population was restricted to Asian patients, which may have led to unexpected selection bias. Secondly, this was a crosssectional study to investigate the baseline among all participants; consequently, it was difficult to establish a causal relationship between OSA and AF.

\section{Conclusions}

The prevalence of AF was in elderly patients with OSA was higher in our study than in similar previous studies. Age, drinking history, CHD, DM, and cardiac dysfunction are dependent risk factors for AF. It is important to determine the risk of AF in elderly patients with OSA because it would reduce the occurrence of major cardiovascular events in these patients. 


\section{Abbreviations}

OSA: obstructive sleep apnea; AF: atrial fibrillation BMI: body mass index; SBP: systolic blood pressure; DBP: diastolic blood pressure; AHI: the apnea-hypopnea index; ODI: the oxygen desaturation index; TST: total sleep time; MSpO2: the mean pulse oxygen saturation; LSpO2: the lowest pulse oxygen saturation;

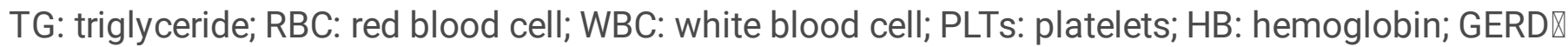
gastroesophageal reflux disease; CHD: coronary heart disease; DM: diabetes mellitus; PVD: peripheral vascular disease; COPD: chronic obstructive pulmonary disease; CKD: chronic kidney disease.

\section{Declarations}

\section{Ethics approval and consent to participate}

The ethics committee of PLA General Hospital approved the study (S2019-352-01).

\section{Consent for publication}

The images and clinical details were agreed to use by all patients in this study. Due to the large number of participants, we telephoned them and all participants agreed orally. And the ethics committee of PLA General Hospital (S2019-352-01) approved the study. The authors agreed to publish this article in magazine.

\section{Availability of data and material}

The datasets used and/or analysed during the current study available from the corresponding author on reasonable request.

\section{Competing interests}

The authors declare no competing financial interests.

\section{Funding}

This study was supported by Open Subject of National Clinical Research Center for Geriatric Diseases (NCRCG-PLAGH-2018008); Military Health Care Project (19BJZ34); Youth Program for Military Medicine of Chinese PLA General Hospital (QNC19054). The funders had not directly role in the design.

\section{Authors' contributions}

Huanhuan Wang, JianHua Li, Yinghui Gao, Kaibing Chen, Yan Gao, JingJing Guo, LiBo Zhao, Xiaofeng Su, Min Shi, Xiao Zou, Weihao Xu, Yabin Wang, Juan Liu, Hu Xu, Junling Lin and Xiaoxuan Kong collected the data. Huanhuan Wang, JianHua Li, and Yinghui Gao analyzed the data and wrote the manuscript draft. Lin Liu, Jiming Han and Xiaoshun Qian designed this study. All the authors contributed to the paper review. 
Acknowledgements

Thanks for every member of this team , and thanks for the support of the Chinese PLA General Hospital fund.

\section{References}

[1] Valenza MC, Baranchuk A, Valenza-Demet G, et al. Prevalence of risk factors for atrial fibrillation and stroke among 1210 patients with sleep disordered breathing. Int J Cardiol. 2014;174(1):73-76.

[2] Khattak HK, Hayat F, Pamboukian SV, et al. Obstructive Sleep Apnea in Heart Failure: Review of Prevalence, Treatment with Continuous Positive Airway Pressure, and Prognosis. Tex Heart Inst J. 2018;45(3):151-161.

[3] Drager LF, McEvoy RD, Barbe F, et al. Sleep Apnea and Cardiovascular Disease: Lessons From Recent Trials and Need for Team Science. Circulation. 2017;136(19):1840-1850.

[4] Ahmad M, Makati D, Akbar S. Review of and Updates on Hypertension in Obstructive Sleep Apnea. Int J Hypertens. 2017;2017:1848375.

[5] Huang T, Lin BM, Stampfer MJ, et al. A Population-Based Study of the Bidirectional Association Between Obstructive Sleep Apnea and Type 2 Diabetes in Three Prospective U.S. Cohorts. Diabetes Care. 2018;41(10):2111-2119.

[6] Takigawa M, Takahashi A, Kuwahara T, et al. Impact of Alcohol Consumption on the Outcome of Catheter Ablation in Patients With Paroxysmal Atrial Fibrillation. J Am Heart Assoc. 2016;5(12):e004149.

[7] Kohno T, Kimura T, Fukunaga K, et al. Prevalence and clinical characteristics of obstructive- and central-dominant sleep apnea in candidates of catheter ablation for atrial fibrillation in Japan. Int $J$ Cardiol. 2018;260:99-102.

[8] Tanaka N, Tanaka K, Hirao Y, et al. Home Sleep Apnea Test to Screen Patients With Atrial Fibrillation for Sleep Apnea Prior to Catheter Ablation. Circ J. 2021;85(3):252-260.

[9] Ben Halima M, Sammoud K, Ben Amar J, et al. Prevalence and predictors of Sleep Apnea in Atrial Fibrillation patients. Tunis Med. 2020;98(12):1031-1038.

[10] Yao Y, Liu F, Wang Y, et al. Lipid levels and risk of new-onset atrial fibrillation: A systematic review and dose-response meta-analysis. Clin Cardiol. 2020;43(9):935-943.

[11] Morales CR, Hurley S, Wick LC, et al. In-home, self-assembled sleep studies are useful in diagnosing sleep apnea in the elderly. Sleep. 2012;35(11):1491-1501. 
[12] Qian X, Yin T, Li T, et al. High levels of inflammation and insulin resistance in obstructive sleep apnea patients with hypertension. Inflammation. 2012;35(4):1507-1511.

[13] Berry RB, Budhiraja R, Gottlieb DJ, et al. Rules for scoring respiratory events in sleep: update of the 2007 AASM Manual for the Scoring of Sleep and Associated Events. Deliberations of the Sleep Apnea Definitions Task Force of the American Academy of Sleep Medicine. J Clin Sleep Med. 2012;8(5):597619.

[14] Kirchhof P, Benussi S, Kotecha D, et al. 2016 ESC Guidelines for the management of atrial fibrillation developed in collaboration with EACTS. Europace. 2016;18(11):1609-1678.

[15] Gutierrez C, Blanchard DG. Diagnosis and Treatment of Atrial Fibrillation. Am Fam Physician. 2016;94(6):442-452.

[16] Hendrikx T, Sundqvist $M$, Sandström $H$, et al. Atrial fibrillation among patients under investigation for suspected obstructive sleep apnea. PLoS One. 2017;12(2):e0171575.

[17] Abumuamar AM, Newman D, Dorian P, et al. Cardiac effects of CPAP treatment in patients with obstructive sleep apnea and atrial fibrillation. J Interv Card Electrophysiol. 2019;54(3):289-297.

[18] Marrugo Pardo G, Romero Moreno LF, Beltrán Erazo P, et al. Respiratory Complications of Adenotonsillectomy for Obstructive Sleep Apnea in the Pediatric Population. Sleep Disord. 2018;2018:1968985.

[19] Riaz S, Bhatti H, Sampat PJ, et al. The Converging Pathologies of Obstructive Sleep Apnea and Atrial Arrhythmias. Cureus. 2020;12(7):e9388.

[20] Healey JS, Amit G, Field TS. Atrial fibrillation and stroke: how much atrial fibrillation is enough to cause a stroke? Curr Opin Neurol. 2020;33(1):17-23.

[21] Hwang $\mathrm{CH}$. Immediate muscle strengthening by an end-effector type gait robot with reduced real-time use of leg muscles: A case series and review of literature.

[22] Rukhadze I, Fenik VB, Benincasa KE, et al. Chronic intermittent hypoxia alters density of aminergic terminals and receptors in the hypoglossal motor nucleus. Am J Respir Crit Care Med.

2010;182(10):1321-1329.

[23] Gallagher C, Hendriks JML, Elliott AD, et al. Alcohol and incident atrial fibrillation - A systematic review and meta-analysis. Int J Cardiol. 2017;246:46-52.

[24] Zhang $\mathrm{H}$, Ruan $\mathrm{H}$, Rahmutula D, et al. Effect of acute and chronic ethanol on atrial fibrillation vulnerability in rats. Heart Rhythm. 2020;17(4):654-660. 
[25] Staszewsky L, Cortesi L, Baviera M, et al. Diabetes mellitus as risk factor for atrial fibrillation hospitalization: Incidence and outcomes over nine years in a region of Northern Italy. Diabetes Res Clin Pract. 2015;109(3):476-484.

[26] Papazoglou AS, Kartas A, Samaras A, et al. Prognostic significance of diabetes mellitus in patients with atrial fibrillation. Cardiovasc Diabetol. 2021;20(1):40.

[27] Huang B, Yang Y, Zhu J, et al. Clinical characteristics and impact of diabetes mellitus on outcomes in patients with nonvalvular atrial fibrillation. Yonsei Med J. 2015;56(1):62-71.

[28] Zhang P, Zhang R, Zhao F, et al. The prevalence and characteristics of obstructive sleep apnea in hospitalized patients with type 2 diabetes in China. J Sleep Res. 2016;25(1):39-46.

[29] Reutrakul S, Mokhlesi B. Obstructive Sleep Apnea and Diabetes: A State of the Art Review. Chest. 2017;152(5):1070-1086.

[30] Khan AA, Lip GYH, Shantsila A. Heart rate variability in atrial fibrillation: The balance between sympathetic and parasympathetic nervous system. Eur J Clin Invest. 2019;49(11):e13174.

\section{Figures}

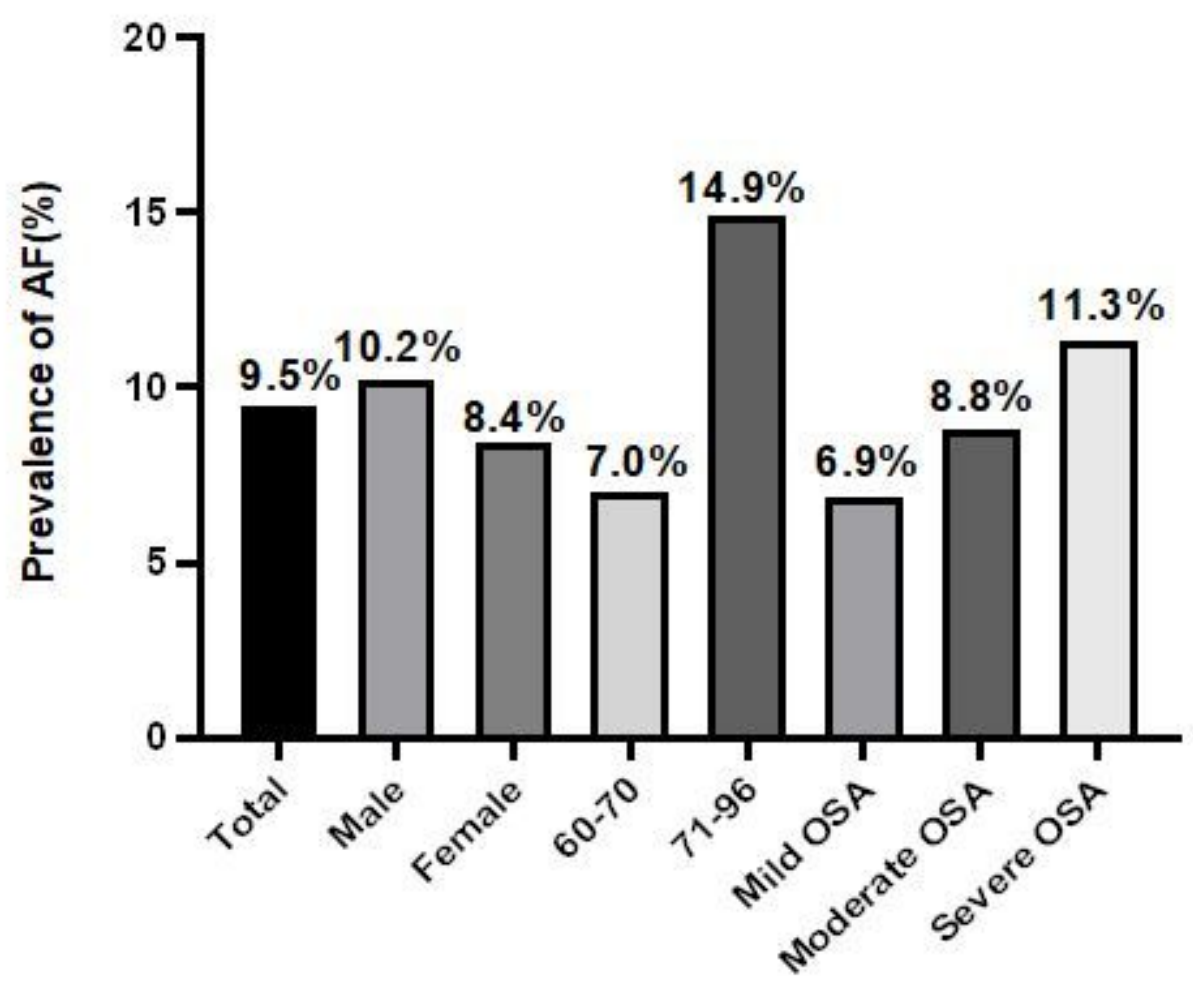

Figure 1 
Prevalence of $A F$ in total study population, male and female, different age groups, and severity of AF classes. AF: atrial fibrillation. OSA: obstructive sleep apnea.

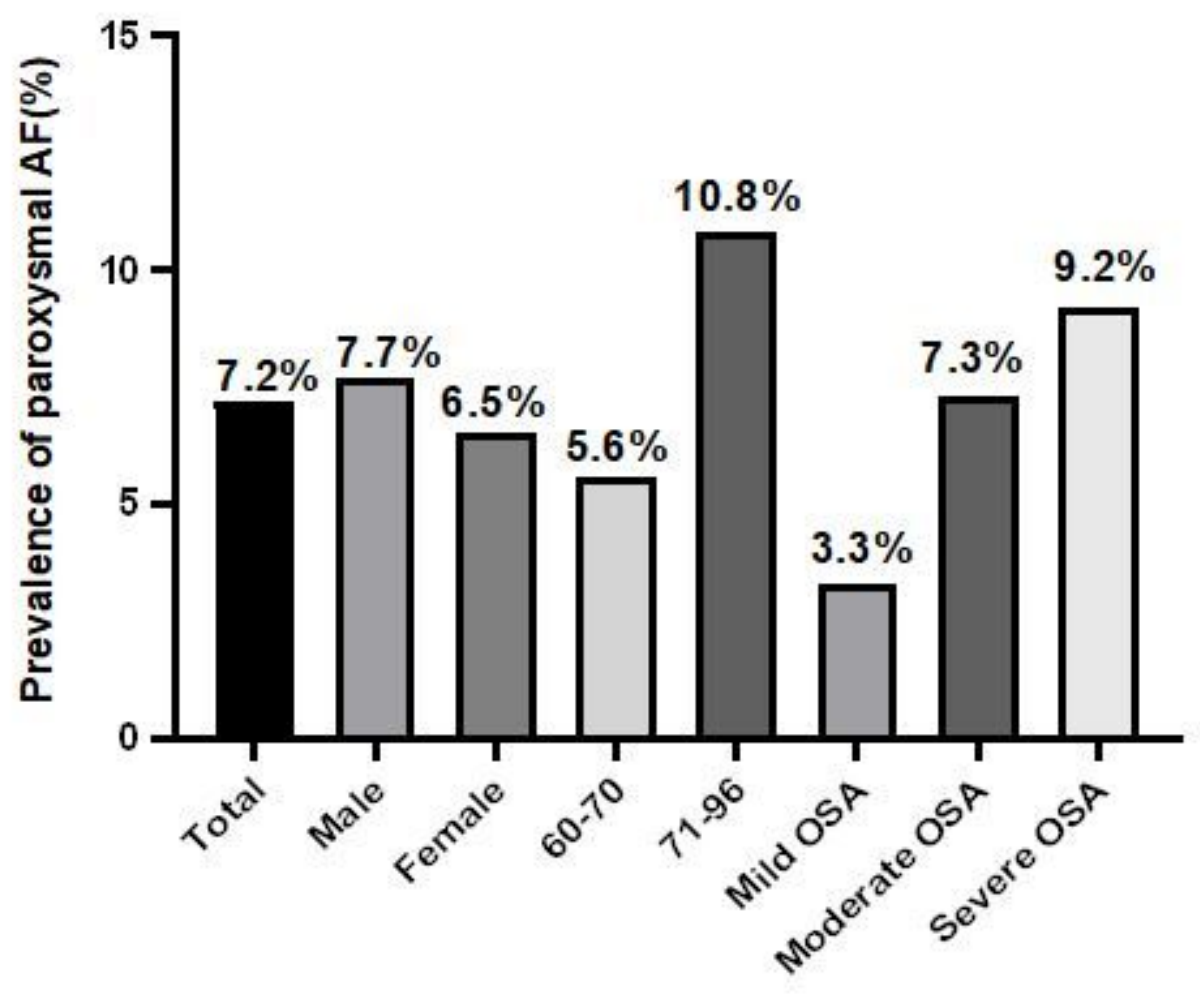

Figure 2

Prevalence of paroxysmal AF in total study population, male and female, different age groups, and severity of AF classes. AF: atrial fibrillation. OSA: obstructive sleep apnea. 


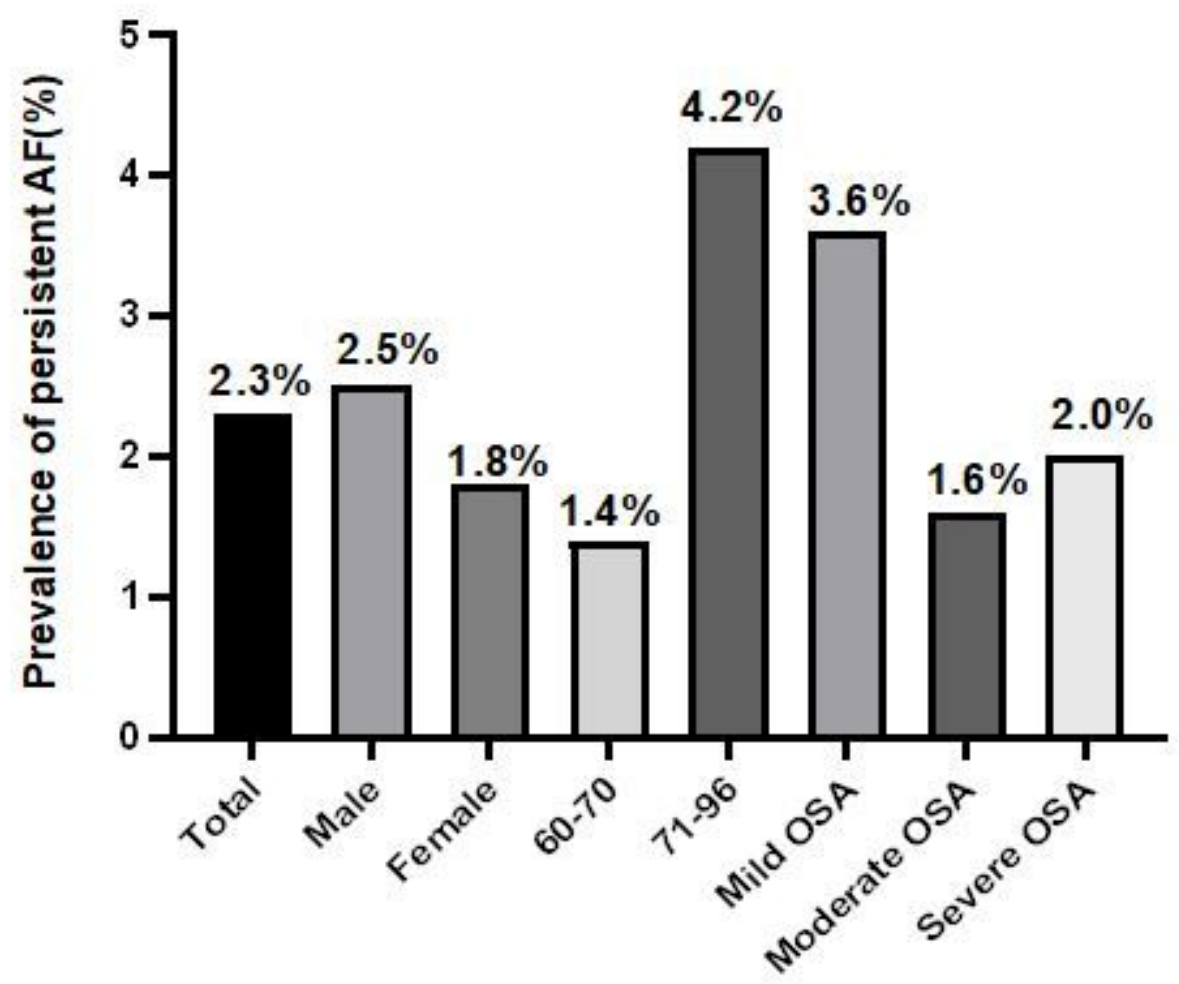

Figure 3

Prevalence of persistent AF in total study population, male and female, different age groups, and severity of AF classes. AF: atrial fibrillation. OSA: obstructive sleep apnea. 